\title{
Generation of subterawatt-attosecond pulses in a soft $x$-ray free-electron laser
}

\author{
Senlin Huang, ${ }^{1, *}$ Yuantao Ding, ${ }^{2, \dagger}$ Zhirong Huang, ${ }^{2}$ and Gabriel Marcus ${ }^{2}$ \\ ${ }^{1}$ Institute of Heavy Ion Physics, School of Physics, Peking University, Beijing 100871, China \\ ${ }^{2}$ SLAC National Accelerator Laboratory, Menlo Park, California 94025, USA
}

(Received 4 February 2016; published 15 August 2016)

\begin{abstract}
We propose a novel scheme to generate attosecond soft x rays in a self-seeded free-electron laser (FEL) suitable for enabling attosecond spectroscopic investigations. A time-energy chirped electron bunch with additional sinusoidal energy modulation is adopted to produce a short seed pulse through a self-seeding monochromator. This short seed pulse, together with high electron current spikes and a cascaded delay setup, enables a high-efficiency FEL with a fresh bunch scheme. Simulations show that using the Linac Coherent Light Source (LCLS) parameters, soft x-ray pulses with a FWHM of 260 attoseconds and a peak power of $0.5 \mathrm{TW}$ can be obtained. This scheme also has the feature of providing a stable central wavelength determined by the self-seeding monochromator.
\end{abstract}

DOI: 10.1103/PhysRevAccelBeams.19.080702

\section{INTRODUCTION}

Over the past few decades, investigations of ultrafast phenomena with ever shorter radiation pulses have paved the way for crucial progress in our understanding of the fundamental processes in matter. For example, timeresolved spectroscopy at the femtosecond time scale has opened a new field in femtochemistry to the study of atomic-scale dynamics of chemical bonds [1]. Extending this technique to the attosecond domain would provide an ideal tool for investigating electronic dynamics on the atomic/molecular scale [2], which are expected to inspire new breakthroughs in ultrafast science $[3,4]$.

Attosecond radiation pulses are currently generated with the high-order harmonic generation (HHG) technique [5,6], which mainly works in the extreme-ultraviolet (XUV) and soft $\mathrm{x}$-ray (up to a few hundred $\mathrm{eV}$ ) spectral regions. Although many important applications of attosecond pulses have been demonstrated, improving the performance of such short pulses is still highly required $[7,8]$. One limitation of the HHG-based radiation source is the relatively low photon flux due to the low conversion efficiency of the harmonic generation process. The highharmonic cutoff also imposes a limit on achieving higher photon energy. Extending the photon energy to the $\mathrm{keV}$ energy level is still a very challenging and exciting topic in the HHG community [9].

\footnotetext{
*huangs1@pku.edu.cn

†ding@slac.stanford.edu
}

Published by the American Physical Society under the terms of the Creative Commons Attribution 3.0 License. Further distribution of this work must maintain attribution to the author $(s)$ and the published article's title, journal citation, and DOI.
X-ray free-electron lasers (FELs) [10,11], which overcome the intensity and photon energy limit of the HHGbased radiation source, provide an alternative way for generating high brightness ultrashort radiation pulses. While a typical $\mathrm{x}$-ray FEL pulse is tens of femtoseconds long, various schemes have been proposed and developed to generate subfemtosecond pulses [12-28]. In a FEL, the radiation slips along the electron bunch in the forward direction and gets amplified. Therefore, a phase correlation inside the slippage distance is formed. This slippage distance is proportional to the cooperation length [29], which determines the temporal spike width (or coherence time) and the shortest achievable pulse. In the hard x-ray spectral region, e.g., at $5 \mathrm{keV}$ or higher, the coherence time is a few hundred attoseconds, while in the soft x-ray region, e.g., $\sim 1 \mathrm{keV}$, it is about $1-2$ fs.

Most of the proposed attosecond FEL schemes leverage the shorter coherence time associated with the production of hard x-rays. However, the plenitude of atomic resonances and efficient photoabsorption in the soft $\mathrm{x}$-ray spectral region has made generating intense attosecond soft x-ray pulses highly desirable by, for instance, the atomic, molecular, and optical (AMO) scientific community [30] and the condensed matter science community [31]. The production of attosecond pulses at soft $\mathrm{x}$-ray FELs is, though, nontrivial. A higher FEL efficiency, namely, a larger $\rho$ [32], would help reduce the coherence time but it requires improving the electron beam brightness. Very recently, two schemes [33,34] of selective lasing with multiple delays have been proposed which are expected to achieve terawatt-level attosecond hard x-ray pulses. In these two schemes, besides taking advantage of the short pulse feature from the enhanced self-amplified spontaneous emission (ESASE) [16] or slotted foil techniques [35], superradiant behavior [36] leveraged to shorten the pulse 
length and increase the power. The involvement of an optical delay in [33] makes the scheme technically very challenging. The naturally longer coherence time in soft x-ray FELs also limits the slotted foil mode in [34] to achieve attosecond soft $x$-ray pulses. In parallel, a few soft $\mathrm{x}$-ray attosecond schemes [37-40] were proposed relying on a coherent emission process with a short undulator to avoid slippage problem, but the output power is relatively low (at $100 \mathrm{~s}$ of Megawatts level).

In this paper, we propose a scheme to generate high intensity attosecond pulses specially for the soft x-ray region. A time-energy chirped electron bunch, which has an additional sinusoidal energy modulation, is used to generate a short seed pulse from a regular soft x-ray selfseeding configuration [41]. This short seed is amplified by one of many high-current spikes that are formed after the self-seeding chicane. The short seed pulse interacting with only a single current spike is a key feature in this scheme, which enables a cascaded delay to compensate the slippage effect and allows further amplification of the seed pulse by adjacent "fresh" current spikes. In this way, both higher FEL efficiency from enhanced current and superradiance with fresh bunch help achieve shorter pulses with higher power. Simulations show that half-terawatt pulses with a duration of $\sim 200$ attoseconds at $830 \mathrm{eV}$ can be generated. In addition, the seeded mode provides a stable central wavelength, which also distinguishes this scheme from the ones in Refs. [33,34].

In the following sections, we will first discuss the methods of the proposed scheme in Sec. II, including the layout and optimization of the parameters. In Sec. III, we discuss the FEL simulation performance. Finally, the results are summarized in Sec. IV.

\section{METHODS}

The proposed layout is shown in Fig. 1. A time-energy chirped electron bunch is used instead of a regular flat-chirp beam. We add an optical modulation section (W) at the very beginning to generate a sinusoidal energy modulation at the optical laser wavelength. This energy-modulated beam then traverses the first section of the undulator (U1) from which a time-frequency chirped self-amplified spontaneous emission (SASE) pulse is generated. As studied earlier [42], a monochromator can be used to select a narrow bandwidth from this chirped radiation pulse, and therefore a short segment of the original radiation can be isolated. In this way, we generate a shorter seed pulse compared to the electron bunch. Meanwhile, the electron beam goes through a bypass chicane and recombines with the seed in the next stage.

The bypass chicane, which exists in self-seeding configuration used for delaying the electron beam and washing out the electron beam microbunching built up in the SASE undulator, now has an additional function. It converts the energy modulation of electron beam into a density modulation at the optical wavelength, forming ESASE-like narrow current spikes with significantly increased peak current [16]. Since the chicane has multiple purposes, we set the magnet strength by satisfying the seeding requirement and then adjust the optical laser intensity to control the current spike.

The FEL amplifier stage after the self-seeding section comprises multiple undulator sections separated by small delay chicanes, which introduce cascaded delay of electron current spikes. At the entrance of the first undulator section (U2A), as illustrated in Fig. 1, the short seed pulse is aligned with the tail current spike. After amplification in the first section, the electron bunch is delayed to align the radiation pulse with the front current spike so that the radiation is continuously amplified by a "fresh" beam. We applied two delay sections in the amplifier stage. The output radiation pulse duration from the FEL amplifier is determined by the length of the interacting part of the electrons and the FEL slippage. Since we have very high current at the spikes, it enables high efficiency FEL gain which reduces the FEL coherence time. The slippage is also compensated by delaying a fresh current spike to overlap with the seed pulse in a new stage.

A time-energy chirped electron beam can be generated by setting the electron beam at an off-crest rf phase in the linac. In this study, we take the overcompressed beam and undulator parameters from the present Linac Coherent

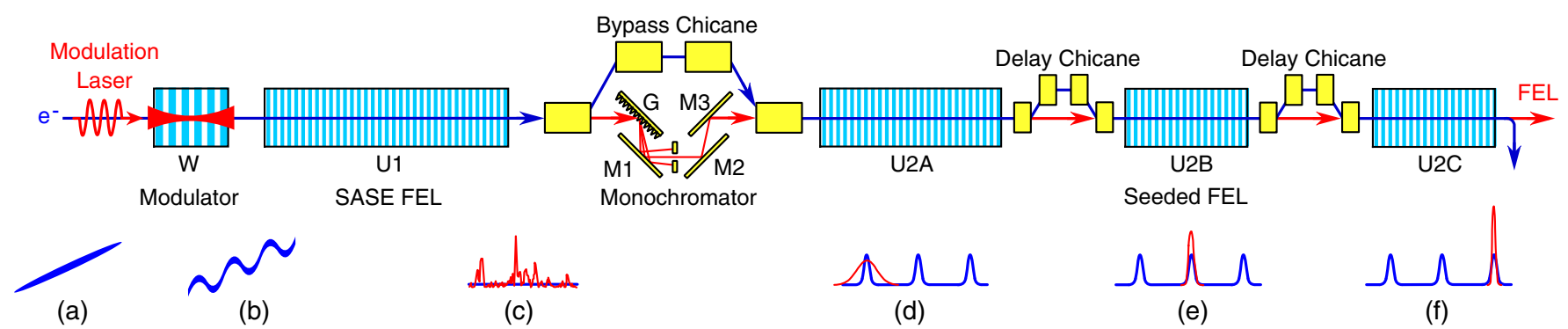

FIG. 1. Layout of the proposed scheme based on a self-seeded FEL configuration (not to scale). A time-energy chirped electron beam (a) is used. A modulator comprising an infrared optical laser and a short wiggler (W) is added to modulate the chirped electron beam (b). The seeded FEL stage consists of three undulator sections (U2A, U2B, and U2C) separated by two delay chicanes. (c), (d), (e), and (f) are sketches of electron current (blue curves) and FEL power (red curves) profiles after U1 and before U2A, U2B, and U2C, respectively. 
Light Source (LCLS) operation [43]. The electron beam central energy $E_{0}$ is $4.3 \mathrm{GeV}$ for generating $1.5 \mathrm{~nm}$ $(830 \mathrm{eV})$ radiation. To be simple, we assume a uniform current profile with a pulse duration of $90 \mathrm{fs}$ and peak current of $2.5 \mathrm{kA}$. The time-energy chirp, $\alpha=\delta / \Delta t$, is $-3.33 \times 10^{-4} \mathrm{fs}^{-1}$ based on the measured data at the LCLS [44], where $\delta=\Delta E / E_{0}$ with $\Delta E$ the correlated rms energy spread. The main parameters used in this study are summarized in Table I.

We follow two criteria to determine the modulation laser parameters. First, the separation between the current spikes should be larger than the seed pulse duration. This is essential in our scheme to generate isolated single pulse. Otherwise satellite low-power peaks would probably show up from the neighboring current spikes. Second, the modulated electron beam should get fully compressed at one side of the zero-crossing phase region after the bypass chicane. Since the chicane strength is predetermined by the self-seeding requirement, we adjust the laser intensity to control the energy modulation strength for getting a full compression of the modulated electrons.

The distances between the neighboring electron current spikes after the bypass chicane can be approximated using

$$
d \approx\left(1+\alpha R_{56} / c\right) \lambda_{m},
$$

where $\lambda_{m}$ is the modulation laser wavelength, and $c$ is the speed of light in vacuum. We consider the laser wavelength $\lambda_{m}$ at $2 \mu \mathrm{m}$ and $5 \mu \mathrm{m}$ here. With the LCLS soft x-ray selfseeding bypass chicane $R_{56}$ about $-0.4 \mathrm{~mm}$ [41], the current spike distances are estimated to be $2.9 \mu \mathrm{m}$ and $7.2 \mu \mathrm{m}$ when $\lambda_{m}$ is $2 \mu \mathrm{m}$ and $5 \mu \mathrm{m}$, respectively. Note here one side of the modulated chirp has the right sign for compression, the other side gets decompressed, and the overall bunch is also decompressed with the chirp sign we have from overcompression mode.

The seed pulse duration after the self-seeding monochromator is mainly determined by the chirp and monochromator bandwidth. This can be calculated using [45]

TABLE I. Main electron and radiation parameters.

\begin{tabular}{lcc}
\hline \hline Parameters & Value & Unit \\
\hline Energy $E_{0}$ & 4.3 & $\mathrm{GeV}$ \\
Time-energy chirp $\alpha$ & $-3.33 \times 10^{-4}$ & $\mathrm{fs}^{-1}$ \\
Slice energy spread $\Delta E_{s}$ & 1 & $\mathrm{MeV}$ \\
Bunch charge $Q$ & 225 & $\mathrm{pC}$ \\
Bunch length FWHM $L_{b}$ & 90 & $\mathrm{fs}$ \\
Peak current $I$ & 2.5 & $\mathrm{kA}$ \\
Normalized emittance $\gamma \epsilon$ & 0.5 & $\mu \mathrm{m}$ \\
Undulator period $\lambda_{u}$ & 3 & $\mathrm{~cm}$ \\
Undulator $K_{u}$ & 3.5 & \\
FEL wavelength $\lambda_{r}$ & 1.5 & $\mathrm{~nm}$ \\
Bypass chicane $R_{56}$ & -0.4 & $\mathrm{~mm}$ \\
Delay chicane $R_{56}$ & -13.2 & $\mu \mathrm{m}$ \\
Mono. resolving power & 5000 & \\
\hline \hline
\end{tabular}

$\sigma_{t}=\sqrt{\frac{\left(\sigma_{\omega} / \omega_{0}\right)^{2}+\left(\sigma_{m} / \omega_{0}\right)^{2}}{4 \alpha^{2}}+\frac{1}{4 \omega_{0}^{2}\left(\sigma_{m} / \omega_{0}\right)^{2}}}$,

where $\sigma_{t}$ is the rms pulse duration after the monochromator, $\sigma_{\omega}$ is the rms bandwidth of the SASE radiation from an unchirped electron beam, $\sigma_{m}$ is the rms bandpass of X-ray monochromator, $\omega_{0}$ is the central SASE frequency. With parameters in Table I, $\omega_{0} \approx 1.24 \times 10^{18} \mathrm{~Hz}, \sigma_{m} / \omega_{0} \approx$ $8.5 \times 10^{-5}$, and $\sigma_{\omega} / \omega_{0} \approx 4.5 \times 10^{-3}$. Consequently, the FWHM of radiation pulse after the monochromator is approximately $6 \mu \mathrm{m}$. From earlier discussion using Eq. (1), a laser with 5- $\mu \mathrm{m}$ wavelength would have a current spike separation of $7.2 \mu \mathrm{m}$, which is larger than the seed pulse duration. Hence a 5- $\mu \mathrm{m}$ laser is an appropriate choice considering the beam chirp we used here to fulfill the first criterion. It is worth pointing out that $\sigma_{\omega} / \omega_{0}$ herein was derived from GENESIS [46] simulations using $\lambda_{m}=5 \mu \mathrm{m}$ as an example. It is significantly increased compared to a typical SASE FEL, owing to the large energy spread of the modulated electron beam.

After determining the modulation laser wavelength, the second criterion, achieving full compression of the modulated electron beam at the zero-crossing phase region, can be met with a peak-to-peak modulation depth of $25.6 \mathrm{MeV}$ with the bypass chicane $R_{56}$ at $-0.4 \mathrm{~mm}$. Based on this requirement, we can determine the optical laser intensity. For example, with a laser at $\lambda_{m}=5 \mu \mathrm{m}$, and using a 5-period wiggler with the period length of $0.35 \mathrm{~m}$ and strength parameter $K_{w}=63.6$, both analytical formula [47] and ELEGANT [48] tracking show the required laser power to be about $35 \mathrm{GW}$. A plot of electron beam longitudinal phase space after the modulator is shown in Fig. 2(a). After passing through the bypass chicane, the
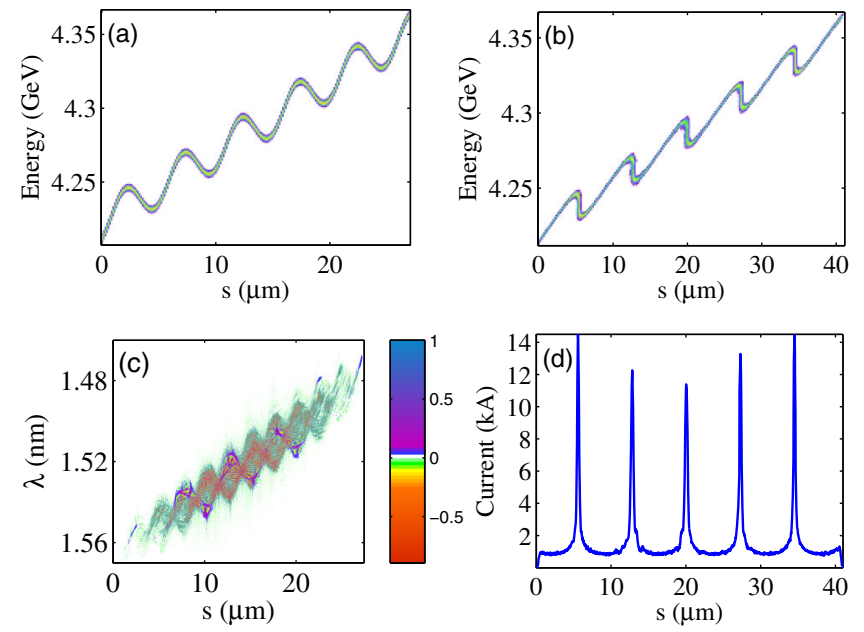

FIG. 2. Electron beam longitudinal phase space before the SASE undulator (a) and after the bypass chicane (b). The Wigner transformation of the SASE field generated in U1 is shown in (c), and the electron current profile after the bypass chicane is show in (d). The electron bunch head is to the right. 
energy modulation is converted to density modulation, as shown in Fig. 2(b). The current profile has over $10 \mathrm{kA}$ spikes as shown in Fig. 2(d).

\section{FEL SIMULATIONS}

Using the modulated electron beam shown in Fig. 2(a) with main parameters listed in Table I, we performed FEL simulations with GENESIS. The SASE stage (U1) uses 5 LCLS undulator segments, each segment having 110 periods with a period length of $3 \mathrm{~cm}$ and strength parameter $K_{u}=3.5$. This is based on a consideration of keeping the SASE FEL power at a reasonably low level to avoid damage to the $\mathrm{x}$-ray monochromator while getting sufficient seed pulse power for the next stage FEL amplifier. We show in Fig. 2(c) a SASE field Wigner distribution function [49] at the end of U1. It is very clear that the radiation field has a time-frequency chirp. This allows us to choose a small fraction in time domain by a narrow-bandpass monochromator. The monochromator, with a resolving power of 5000, is assumed to have a Gaussian spectral response with a maximum power efficiency of 0.02 at $1.5 \mathrm{~nm}$ in our simulations.

After this SASE field passing through the monochromator, a seed pulse is generated as shown in Fig. 3(a) with a red curve. The seed pulse duration-about $6 \mu \mathrm{m}$ FWHM-is consistent with the estimation from Eq. (2). We apply a fine adjustment of the bypass chicane here to align this short seed with the tail current spike (the alignment timing jitter will be discussed later). This adjustment of the bypass chicane is only a few percentage of the existing delay for seeding requirement, and should not affect the electron beam current distribution. The FEL amplifier consists of 3 undulator sections (U2A, U2B, and U2C in Fig. 1) separated by 2 small delay chicanes with $R_{56} \approx-13.2 \mu \mathrm{m}$, which introduce electron delay of about $6.6 \mu \mathrm{m}$. Among the 3 undulator sections, U2A, U2B, and U2C use 4,2 , and 3 LCLS undulator segments, respectively. In each amplifier section, we optimize the undulator length to have sufficient gain from the interaction between the seed pulse and the selected electron current spike, while at the same time to mitigate the FEL slippage effect and the SASE growth in other neighboring spikes. The delay chicanes realign a front "fresh" current spike to the high-power seed and also help wash out some of the electron beam microbunching generated in the previous undulator, which will benefit the next stage FEL amplification.

The high peak current in these spikes leads to a strong longitudinal space charge (LSC) force, which will accelerate/decelerate the head/tail part of electrons in the spike area [50]. In the undulator, this LSC force is stronger compared to that in a drift space due to the wiggling motion [51]. We include this effect in the FEL simulations, and taper the undulator strength to compensate the LSC induced energy chirp $[17,19]$. In U2A, the energy chirp caused by the LSC force is still low. The undulator parameter is therefore kept unchanged at $K_{u}=3.5$. In $\mathrm{U} 2 \mathrm{~B}$ and $\mathrm{U} 2 \mathrm{C}$, however, after interaction with the electrons for a longer distance, the LSC force leads to a distinct timeenergy chirp in the current spikes. To preserve the resonant condition as the radiation slips toward the high energy head of the current spike, the $K_{u}$ values of $\mathrm{U} 2 \mathrm{~B}$ and $\mathrm{U} 2 \mathrm{C}$ are linearly increased (reversely tapered) by $0.1 \%$ and $0.225 \%$ per meter, respectively.

Plots in Fig. 3 illustrate the evolution of the FEL power profiles and electron energy spread along the FEL amplifier. We can see that, as the electron beam goes through $\mathrm{U} 2 \mathrm{~A}, \mathrm{U} 2 \mathrm{~B}$, and $\mathrm{U} 2 \mathrm{C}$, only the electrons within the interacting current spike show an increase of the energy spread. This indicates that the FEL has been successfully amplified within the overlapped spike area, and other current spikes are relatively "fresh." One can also see from the figure that, after $\mathrm{U} 2 \mathrm{~A}$, the radiation pulse becomes much shorter as expected [see subplot (b)], since only a portion of the radiation interacts with the electrons within the current spike. After $\mathrm{U} 2 \mathrm{~B}$ and $\mathrm{U} 2 \mathrm{C}$, the radiation pulse duration gets further reduced, reaching 260 attoseconds (FWHM), with a power of over $800 \mathrm{GW}$ in this example.
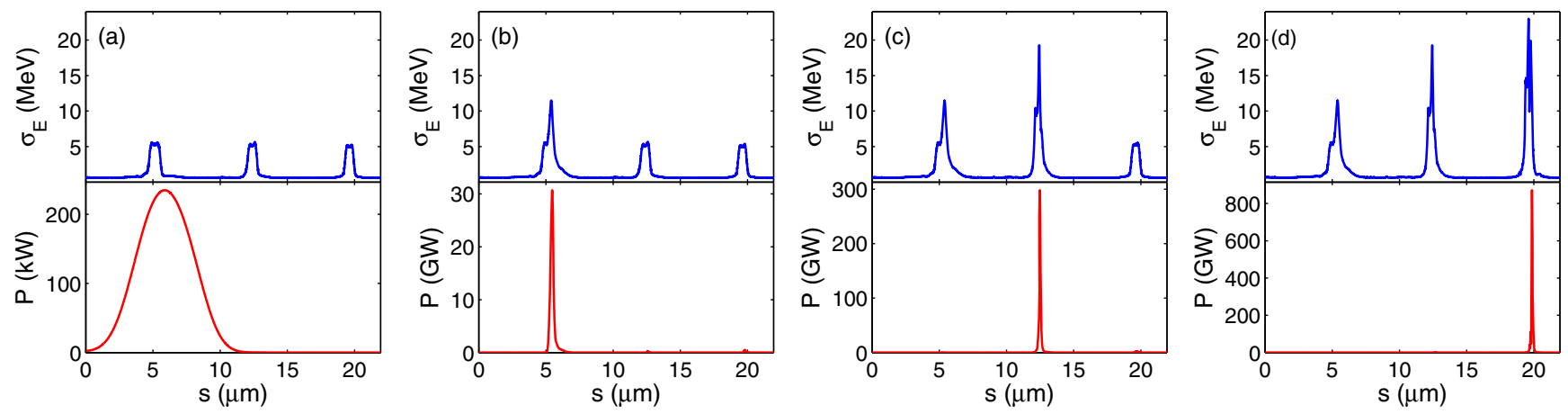

FIG. 3. Simulation results of rms electron energy spread (upper) and FEL power profile (lower) right after the bypass chicane (a) and U2A (b), U2B (c), and U2C (d) undulators. The electron bunch head is to the right. The three high energy spread regions in (a) correspond to the three currents spikes from the center to the right in Fig. 2(d). 

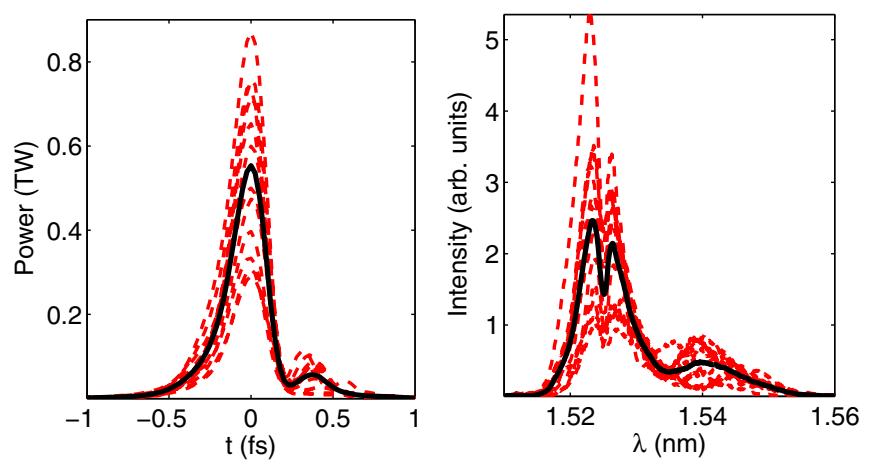

FIG. 4. Multiple-shot simulation results of FEL power profile (left) and spectrum (right) at the end of U2C undulator. The solid curves in the plots are the average over all shots.

To investigate the statistical fluctuation on the radiation pulses, multiple GENESIS runs have been performed with different random shot noise initialization in the SASE stage. Figure 4(a) shows 10 shots of the simulated final FEL power profiles and spectra. Simulation shows that there is always one dominate single spike in the output $\mathrm{x}$-ray pulses. These shots have an average peak power of $550 \pm 200 \mathrm{GW}$, and an average pulse duration of $260 \pm 13$ attoseconds. The average spectrum for these shots is centered at $1.53 \mathrm{~nm}$ with a FWHM bandwidth of about $0.5 \%$.

In the above discussions, the system is set in an ideal condition. That is, the timing between the modulation laser and the electron bunch and the delay of the electron beam in the chicanes are properly optimized. For a practical operation, however, jitter could affect the performance in this scheme. Here the main jitter source includes the electron beam energy jitter and the timing jitter between the electron beam and the modulation laser. The photon energy is determined by the monochromator, and electron beam energy jitter mainly affect the timing after passing through chicanes. For example, with a typical energy jitter at the $10^{-4}$ level, it converts to timing jitter of about $0.1 \mathrm{fs}$ after the self-seeding bypass chicane. This is relatively small compared to the seed pulse duration ( $\sim 20 \mathrm{fs})$. Thus the main concern in this scheme will be the timing jitter between the optical modulation laser and the electron bunch.

An achievable timing jitter between the electron beam and the modulation laser is at the 20 -fs level, as reported in [52]. Since the modulation laser is assumed to be much longer than the electron bunch, they can easily overlap in time. So the overall timing between them is not a problem. However, after the bypass chicane, this timing jitter will be converted to current spike position jitter (the current spike is shifting around along the bunch). While the seed pulse, determined by the beam chirp and the monochromator, stays at a fixed longitudinal position with respect to the electron bunch. Thus the current spike position jitter will cause a misalignment between the seed pulse and current spike. Based on the parameters discussed in this paper, the duration of seed pulse from the monochromator is $\sigma_{s} \approx 2.5 \mu \mathrm{m}$, and the spacing between the current spikes is $\Delta s=7.2 \mu \mathrm{m}$. If we require the acceptable alignment to be within $\pm \sigma_{s}$, the ratio of the "good" aligned shots would be $\sim 70 \%$ assuming the current spike is a delta function. The small delay chicanes should be always set at a fixed delay value determined by the spacing of the current spikes, thus we only need to consider the timing jitter in the first amplifier stage (U2A). Also note that the modulation is periodic, so the value of the timing jitter does not affect the ratio of good shots as long as they are overlapped.

Comparing to the results with a perfect alignment shown in Fig. 4, how the FEL performance will be affected with $\pm \sigma_{s}$ misalignment? One could imagine that some lowintensity satellite radiation spikes will come up because the seed pulse may cover two spikes due to the misalignment. In our study, we noticed that this is true in the early amplifier stages, but the cascaded delay actually helps suppress the satellite spikes in the following stages. We show this delay-enabled selecting process in Fig. 5. A misalignment of 8 fs was set in this example. In the U2A stage, we have two pulses with one of them dominant. Then after delaying both of them to fresh spikes, the dominant high-power pulse will grow faster. Finally we can still achieve a single-spike FEL pulse with high power.
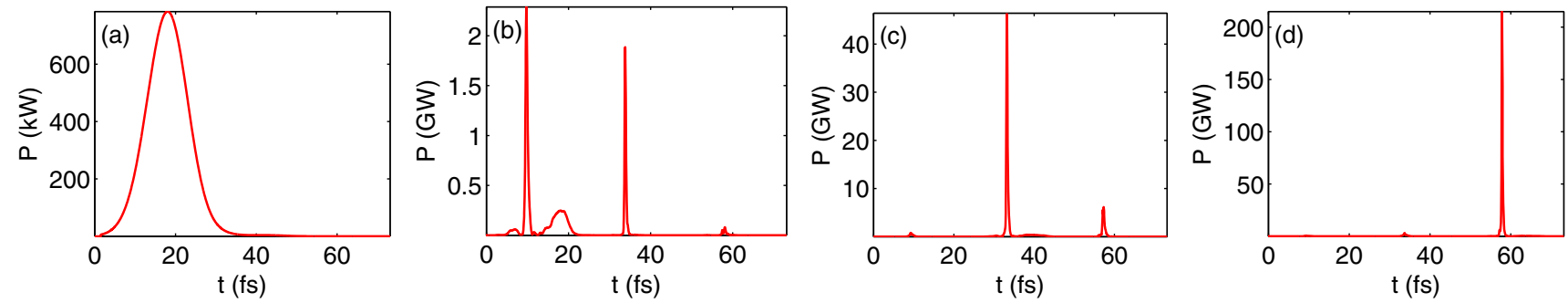

FIG. 5. An example of FEL power profiles at U2A entrance (a), U2A exit (b), U2B exit (c), and U2C exit (d) with a misalignment of $8 \mathrm{fs}$ between the modulation laser and the electron bunch. Multi-spikes appear at the first amplifier stage (U2A), but the cascaded delay helps select the dominant spike. 


\section{SUMMARY}

In summary, we have presented a scheme for generating attosecond soft x-ray pulses in a self-seeded FEL mode using a modulated chirped electron beam. Numerical study has been carried out to demonstrate this scheme with the LCLS parameters. At $1.5 \mathrm{~nm}$ wavelength, simulation shows X-ray pulses with a FWHM of approximately 260 attoseconds and a peak power of a few hundred gigawatts can be obtained. This scheme also has the feature of providing a stable central wavelength which is determined by the selfseeding monochromator. Also note the modulation laser could provide a reference signal for the pump-probe experiments in the x-ray hutch. As discussed, the timing jitter should be considered during operation. A delayenabled selecting process helps suppress the side peaks, and we expect more than half of the shots to be single spike considering a random jitter between the optical modulation laser and the electron beam.

The electron beam chirp used in this study is based on the present measured beam with over-compression mode at the LCLS [44]. With this chirp and the present LCLS soft $\mathrm{x}$-ray monochromator, here we chose a 5- $\mu \mathrm{m}$ wavelength optical laser. A larger chirp would be possible to allow shorter wavelength laser such as at $2 \mu \mathrm{m}$. A recent dechirper device using corrugated structure at the LCLS has been commissioned and would help increase the chirp in the over-compression mode [53,54]. This chirp control provides more flexibilities on choosing the laser parameters for this scheme.

\section{ACKNOWLEDGMENTS}

We thank A. Marinelli and R. Coffee for helpful discussions. This work is partially supported by the U.S. Department of Energy Contract No. DE-AC0276 SF00515.

[1] A. H. Zewail, Femtochemistry: Atomic-scale dynamics of the chemical bond, J. Phys. Chem. A 104, 5660 (2000).

[2] Z. Chang and P. Corkum, Attosecond photon sources: the first decade and beyond, J. Opt. Soc. Am. B 27, B9 (2010).

[3] P. H. Bucksbaum, The future of attosecond spectroscopy, Science 317, 766 (2007).

[4] F. Krausz and M. Ivanov, Attosecond physics, Rev. Mod. Phys. 81, 163 (2009).

[5] X. F. Li, A. L'Huillier, M. Ferray, L. A. Lompré, and G. Mainfray, Multiple-harmonic generation in rare gases at high laser intensity, Phys. Rev. A 39, 5751 (1989).

[6] A. McPherson, G. Gibson, H. Jara, U. Johann, T. S. Luk, I. A. McIntyre, K. Boyer, and C. K. Rhodes, Studies of multiphoton production of vacuum-ultraviolet radiation in the rare gases, J. Opt. Soc. Am. B 4, 595 (1987).

[7] G. Sansone, L. Poletto, and M. Nisoli, High-energy attosecond light sources, Nat. Photonics 5, 655 (2011).
[8] M. Chini, K. Zhao, and Z. Chang, The generation, characterization and applications of broadband isolated attosecond pulses, Nat. Photonics 8, 178 (2014).

[9] T. Popmintchev et al., Bright coherent ultrahigh harmonics in the kev $\mathrm{x}$-ray regime from mid-infrared femtosecond lasers, Science 336, 1287 (2012).

[10] Z. Huang and K.-J. Kim, Review of x-ray free-electron laser theory, Phys. Rev. ST Accel. Beams 10, 034801 (2007).

[11] B. W. J. McNeil and N. R. Thompson, X-ray free-electron lasers, Nat. Photonics 4, 814 (2010).

[12] E. Hemsing, G. Stupakov, D. Xiang, and A. A. Zholents, Beam by design: Laser manipulation of electrons in modern accelerators, Rev. Mod. Phys. 86, 897 (2014).

[13] E. L. Saldin, E. A. Schneidmiller, and M. V. Yurkov, Scheme for attophysics experiments at a X-ray SASE FEL, Opt. Commun. 212, 377 (2002).

[14] E. L. Saldin, E. A. Schneidmiller, and M. V. Yurkov, Terawatt-scale sub-10-fs laser technology-key to generation of GW-level attosecond pulses in X-ray free electron laser, Opt. Commun. 237, 153 (2004).

[15] E. L. Saldin, E. A. Schneidmiller, and M. V. Yurkov, Terawatt-scale sub-10-fs laser technology-key to generation of GW-level attosecond pulses in X-ray free electron laser, Opt. Commun. 239, 161 (2004).

[16] A. A. Zholents, Method of an enhanced self-amplified spontaneous emission for x-ray free electron lasers, Phys. Rev. ST Accel. Beams 8, 040701 (2005).

[17] E. L. Saldin, E. A. Schneidmiller, and M. V. Yurkov, Selfamplified spontaneous emission FEL with energy-chirped electron beam and its application for generation of attosecond x-ray pulses, Phys. Rev. ST Accel. Beams 9, 050702 (2006).

[18] A. A. Zholents and M. S. Zolotorev, Attosecond x-ray pulses produced by ultra short transverse slicing via laser electron beam interaction, New J. Phys. 10, 025005 (2008).

[19] Y. Ding, Z. Huang, D. Ratner, P. Bucksbaum, and H. Merdji, Generation of attosecond x-ray pulses with a multicycle two-color enhanced self-amplified spontaneous emission scheme, Phys. Rev. ST Accel. Beams 12, 060703 (2009).

[20] J. Qiang and J. Wu, Generation of multi-color attosecond $\mathrm{X}$-ray radiation through modulation compression, Appl. Phys. Lett. 99, 081101 (2011).

[21] J. Qiang and J. Wu, Generation of attosecond coherent $\mathrm{X}$-ray radiation through modulation compression, J. Mod. Opt. 58, 1452 (2011).

[22] P. Emma, Z. Huang, and M. Borland, Attosecond X-ray pulses in the LCLS using the slotted foil method, in Proceedings of FEL2004 Conference (Comitato Conferenze Elettra, Trieste, Italy, 2004), p. 333.

[23] J. B. Rosenzweig et al., Generation of ultra-short, high brightness electron beams for single-spike SASE FEL operation, Nucl. Instrum. Methods Phys. Res., Sect. A 593, 39 (2008).

[24] S. Reiche, P. Musumeci, C. Pellegrini, and J. B. Rosenzweig, Development of ultra-short pulse, single coherent spike for SASE x-ray FELs, Nucl. Instrum. Methods Phys. Res., Sect. A 593, 45 (2008). 
[25] V. Wacker et al., Sub-femtosecond hard x-ray pulse from very low charge beam at LCLS, in Proceedings of FEL2012 (JACoW, Nara, Japan, 2012), p. 606.

[26] S. Huang, Y. Ding, Z. Huang, and J. Qiang, Generation of stable subfemtosecond hard X-ray pulses with optimized nonlinear bunch compression, Phys. Rev. ST Accel. Beams 17, 120703 (2014).

[27] N. R. Thompson and B. W. J. McNeil, Mode Locking in a Free-Electron Laser Amplifier, Phys. Rev. Lett. 100, 203901 (2008).

[28] D. J. Dunning, B. W. J. McNeil, and N. R. Thompson, Few-Cycle Pulse Generation in an X-Ray Free-Electron Laser, Phys. Rev. Lett. 110, 104801 (2013).

[29] R. Bonifacio, L. De Salvo, P. Pierini, N. Piovella, and C. Pellegrini, Spectrum, Temporal Structure, and Fluctuations in a High-Gain Free-Electron Laser Starting from Noise, Phys. Rev. Lett. 73, 70 (1994).

[30] C. Bostedt et al., Ultra-fast and ultra-intense x-ray sciences: first results from the Linac Coherent Light Source freeelectron laser, J. Phys. B 46, 164003 (2013).

[31] R. W. Schoenlei et al., New science opportunities enabled by LCLS-II x-ray lasers, SLAC Report No. SLAC-R-1053, 2015.

[32] R. Bonifacio, C. Pellegrini, and L. Narducci, Collective instabilities and high-gain regime in a free electron laser, Opt. Commun. 50, 373 (1984).

[33] T. Tanaka, Proposal for a Pulse-Compression Scheme in X-Ray Free-Electron Lasers to Generate a Multiterawatt, Attosecond X-Ray Pulse, Phys. Rev. Lett. 110, 084801 (2013).

[34] E. Prat and S. Reiche, Simple Method to Generate Terawatt-Attosecond X-Ray Free-Electron-Laser Pulses, Phys. Rev. Lett. 114, 244801 (2015).

[35] P. Emma, K. Bane, M. Cornacchia, Z. Huang, H. Schlarb, G. Stupakov, and D. Walz, Femtosecond and Subfemtosecond X-Ray Pulses from a Self-Amplified SpontaneousEmission-Based Free-Electron Laser, Phys. Rev. Lett. 92, 074801 (2004).

[36] R. Bonifacio, L. D. Salvo, P. Pierini, and N. Piovella, The superradiant regime of a FEL: Analytical and numerical results, Nucl. Instrum. Methods Phys. Res., Sect. A 296, 358 (1990).

[37] A. A. Zholents and W. M. Fawley, Proposal for Intense Attosecond Radiation from an X-Ray Free-Electron Laser, Phys. Rev. Lett. 92, 224801 (2004).

[38] D. Xiang, Z. Huang, and G. Stupakov, Generation of intense attosecond $\mathrm{X}$-ray pulses using ultraviolet laser induced microbunching in electron beams, Phys. Rev. ST Accel. Beams 12, 060701 (2009).

[39] A. Zholents and G. Penn, Generation of intense attosecond $\mathrm{X}$-ray pulses using ultraviolet laser induced microbunching in electron beams, Nucl. Instrum. Methods Phys. Res., Sect. A 612, 254 (2010).

[40] J. Yan, H. Deng, D. Wang, and Z. Dai, EEHG-assisted FEL schemes for attosecond X-ray pulses generation, Nucl. Instrum. Methods Phys. Res., Sect. A 621, 97 (2010).

[41] D. Ratner et al., Experimental Demonstration of a Soft X-Ray Self-Seeded Free-Electron Laser, Phys. Rev. Lett. 114, 054801 (2015).

[42] C. B. Schroeder, C. Pellegrini, S. Reiche, J. Arthur, and P. Emma, Chirped-beam two-stage free-electron laser for high-power femtosecond x-ray pulse generation, J. Opt. Soc. Am. B 19, 1782 (2002).

[43] P. Emma et al., First lasing and operation of an angstromwavelength free-electron laser, Nat. Photonics 4, 641 (2010).

[44] C. Emma, Y. Ding, Z. Huang, A. A. Lutman, G. Marcus, A. Marinelli, and C. Pellegrini, Femtosecond X-ray pulse generation with an energy chirped electron beam, in Proceedings of FEL2015 (JACoW, Daejeon, Korea, 2015), p. 722.

[45] S. Krinsky and Z. Huang, Frequency chirped self-amplified spontaneous-emission free-electron lasers, Phys. Rev. ST Accel. Beams 6, 050702 (2003).

[46] S. Reiche, Frequency chirped self-amplified spontaneousemission free-electron lasers, Nucl. Instrum. Methods Phys. Res., Sect. A 429, 243 (1999).

[47] A. A. Zholents and G. Penn, Obtaining attosecond x-ray pulses using a self-amplified spontaneous emission free electron laser, Phys. Rev. ST Accel. Beams 8, 050704 (2005).

[48] M. Borland, Elegant: A flexible SDDS-compliant code for accelerator simulation, Advanced Photon Source Report No. LS-287, 2000.

[49] E. Wigner, On the quantum correction for thermodynamic equilibrium, Phys. Rev. 40, 749 (1932).

[50] A. W. Chao, Physics of Collective Beam Instabilities in High Energy Accelerators (Wiley, New York, 1993).

[51] G. Geloni, Longitudinal impedance and wake from XFEL undulators. Impact on current-enhanced SASE schemes, Nucl. Instrum. Methods Phys. Res., Sect. A 583, 228 (2007).

[52] S. Schulz et al., Femtosecond all-optical synchronization of an X-ray free-electron laser, Nat. Commun. 6, 5938 (2015).

[53] Z. Zhang, K. Bane, Y. Ding, Z. Huang, R. Iverson, T. Maxwell, G. Stupakov, and L. Wang, Electron beam energy chirp control with a rectangular corrugated structure at the Linac Coherent Light Source, Phys. Rev. ST Accel. Beams 18, 010702 (2015).

[54] M. W. Guetg et al., Commissioning of the RadiaBeam/ SLAC dechirper, in Proceedings of IPAC2016 (JACoW, Busan, Korea, 2016), p. 809. 